\title{
Narcissism in Midlife: Longitudinal Changes in and Correlates of Women's Narcissistic Personality Traits
}

\author{
Robin S. Edelstein, Nicola J. Newton, and \\ Abigail J. Stewart \\ University of Michigon
}

\begin{abstract}
We examined changes in and correlates of 3 kinds of narcissism-hypersensitivity, willfulness, and autonomy - during middle adulthood. Few studies have examined narcissistic personality traits beyond young adulthood, and none has assessed longitudinal changes in narcissism during midlife. In a sample of 70 college-educated women, we found that observer ratings of hypersensitive narcissism were associated with more negative outcomes at ages 43 and 53 (i.e., more depressive symptoms and physical health problems, lower life satisfaction and wellbeing). Ratings of willfulness and autonomy predicted more positive outcomes. All 3 kinds of narcissism showed considerable rank-order stability over 10 years, but there were also mean-level changes: Hypersensitivity and autonomy decreased, whereas willfulness increased. More positive outcomes were associated with decreases in hypersensitivity and increases in willfulness and autonomy. However, in multivariate analyses, autonomy did not show any significant associations with women's

Nicola J. Newton is now in the School of Education and Social Policy at Northwestern University.

Collection of the data reported in this research has been supported by the Boston University Graduate School, National Science Foundation Visiting Professorships for Women, the Society for the Psychological Study of Social Issues, the MacArthur Foundation Network for Research on Successful Midlife Development, Radcliffe Research Support and Midlife Program Grants from the Henry A. Murray Research Center, National Institute of Mental Health subgrants under Grants 1-RO1-MH43948 and 1-RO1-MH47408, National Institute on Aging Training Grant T32-AG0017, and the University of Michigan. Computer-accessible and other data from several previous waves are archived at the Henry A. Murray Research Center, Radcliffe College, 10 Garden Street, Cambridge, MA 01238.

Correspondence concerning this article should be addressed to Robin S. Edelstein, Department of Psychology, University of Michigan, 530 Church Street, Ann Arbor, MI 48109. Email: redelste@umich.edu.
\end{abstract}

Journal of Personality 80:5, October 2012

(C) 2011 The Authors

Journal of Personality (C) 2011, Wiley Periodicals, Inc.

DOI: $10.1111 / \mathrm{j} .1467-6494.2011 .00755 . x$ 
health and well-being outcomes, suggesting that it may have less predictive utility compared to hypersensitivity and willfulness. Our findings highlight developmental changes in and correlates of women's narcissistic personality traits and the importance of assessing different aspects of narcissism in midlife.

Middle adulthood has been described as a time of both psychological growth and increasing awareness of limitations (Lachman, 2004). On the one hand, there is evidence for increases in confidence, agency, warmth, and generativity during this period (e.g., Helson \& Wink, 1992; Stewart, Ostrove, \& Helson, 2001). Rather than being fixed in early adulthood, personality clearly continues to change throughout midlife, and many of these changes are in the direction of positive growth and development (e.g., Roberts, Walton, \& Viechtbauer, 2006). At the same time, with middle adulthood comes an increased awareness of aging, physical decline, and the limitations imposed by mortality (Greenberg, 2009). Thus, for some, the transition to middle adulthood may bring an increased sense of empowerment, security, and personal growth (Stewart et al., 2001), but others may struggle with regret and the realization of lost opportunities (Kohut, 1977; Stewart \& Vandewater, 1999).

In the current study, we investigated this life transition, from age 43 to 53, in a longitudinal sample of 70 college-educated women. We were particularly interested in how women's adjustment during this period would be influenced by individual differences in narcissism, a personality construct marked by feelings of self-importance, grandiosity, and entitlement. From a theoretical perspective, narcissistic individuals should have difficulty coping with the heightened sense of limitations and lost opportunities brought on by the transition to middle adulthood (Greenberg, 2009; Kernberg, 1975). However, because the vast majority of empirical work on narcissism has relied on young adult samples, relatively little is known about narcissism in older age groups. Moreover, there are virtually no longitudinal data on changes in narcissism over time (Roberts, Edmonds, \& Grijalva, 2010). The women in our sample, graduates of the Radcliffe College class of 1964, have been followed for over 40 years (e.g., Stewart \& Vandewater, 1993), and observer ratings of narcissism are available from their assessments in 1986 and 1996. Although these women are highly educated and demographically homogeneous, they provide a rare opportunity to study changes in narcissism during middle adulthood. 
We also explored whether different kinds of narcissism predicted different health and well-being outcomes for women during this transition. Although narcissism encompasses a number of maladaptive and even pathological personality attributes, such as grandiosity and entitlement (Miller et al., 2011; Russ, Shedler, Bradley, \& Westen, 2008), it also includes several more adaptive attributes, such as autonomy and self-confidence (Wink, 1992a). These distinctions are captured in Wink's (1992a) conceptualization of three kinds of narcissism-hypersensitivity, willfulness, and autonomy. Because hypersensitivity includes the most pathological characteristics (e.g., defensiveness; Wink, 1992b), and because some have argued that hypersensitive narcissism may be particularly salient or influential among women (e.g., O'Leary \& Wright, 1986), we expected that this dimension of narcissism would be the most consistent predictor of poor health and well-being as women transitioned to middle adulthood.

Finally, we examined changes in women's narcissism during this period and the extent to which such changes predicted health and well-being. There are reasons to expect decreases in narcissism throughout adulthood (Roberts et al., 2010), particularly for pathological aspects of narcissism such as hypersensitivity (Kohut, 1971), and cross-sectional research provides some evidence for age-related declines (Foster, Campbell, \& Twenge, 2003; Ronningstam, Gunderson, \& Lyons, 1995). However, to our knowledge, there have been no longitudinal investigations of narcissism in middle adulthood. In the current study, we expected that women's levels of narcissism, particularly hypersensitivity, would decrease over time and that decreases in hypersensitivity would be associated with better health and well-being.

\section{Adaptive and Maladaptive Aspects of Narcissism}

Narcissism is characterized by arrogance, feelings of superiority and grandiosity, a sense of entitlement, lack of empathy, and interpersonally exploitive behavior (Emmons, 1984; Kernberg, 1975). This constellation of personality attributes can be manifested both as a clinical disorder (narcissistic personality disorder [NPD]; American Psychiatric Association, 1994) and as a normally distributed personality characteristic (Miller \& Campbell, 2010; Raskin \& Hall, 1979). At first blush, narcissistic traits appear maladaptive, and indeed both NPD and narcissistic traits have been associated with impaired interpersonal functioning and psychological maladjustment (e.g., Miller, 
Campbell, \& Pilkonis, 2007). Upon closer examination, however, the picture is more complex: Narcissistic traits also predict many positive outcomes, including higher levels of self-esteem, happiness, and wellbeing (e.g., Zuckerman \& O'Loughun, 2009).

These contradictory findings can be explained, at least in part, by the multifaceted nature of narcissism. That is, narcissism is thought to include both adaptive and maladaptive components (Kernberg, 1975; Kohut, 1971), with NPD reflecting particularly high levels of maladaptive narcissism (Pincus \& Lukowitsky, 2010). In general, there is emerging consensus that two primary dimensions underlie the maladaptive or pathological manifestations of narcissism and NPD: grandiosity and vulnerability (Cain, Pincus, \& Ansell, 2008; Miller et al., 2011; Wink, 1991b). These two dimensions of narcissism share in common deep-seated feelings of self-importance and entitlement, but they differ in their outward expression: Grandiose narcissism is associated with greater assertiveness, exhibitionism, and manipulativeness, whereas vulnerable narcissism is associated with an apparent lack of confidence, shyness, defensiveness, and hypersensitivity. Support for this distinction has also been found among individuals meeting official (DSM-IV; American Psychiatric Association, 1994) criteria for NPD (Russ et al., 2008), although $D S M-I V$ criteria generally emphasize grandiose over vulnerable aspects of this disorder (Cain et al., 2008). Both aspects of pathological narcissism have a number of negative correlates, but vulnerable narcissism has generally been associated with more maladaptive outcomes compared to grandiose narcissism (e.g., Ackerman et al., 2011), including in clinical populations (Russ et al., 2008).

Aspects of narcissism that are thought to be "healthier" or less pathological include autonomy, leadership, and social potency. For instance, the most widely used self-report measure of narcissism, the Narcissistic Personality Inventory (NPI; Raskin \& Hall, 1979), includes a subscale that is typically labeled "Leadership/Authority" (L/A). L/A is associated with more positive qualities (e.g., selfcontrol) and fewer negative qualities (e.g., aggression) compared to other, less adaptive NPI subscales (e.g., Ackerman et al., 2011; Emmons, 1984; Rosenthal \& Hooley, 2010). Even among NPD patients, those who exhibit more adaptive features of narcissism (e.g., achievement orientation) show better overall functioning compared to those exhibiting more pathological features (e.g., lack of empathy; Russ et al., 2008). 
Based on theoretical distinctions and DSM-III criteria, Wink (1992a) developed measures of three types of narcissistic personality traits, which he termed hypersensitivity, willfulness, and autonomy. There are clear differences among the dimensions, but all three are positively correlated with observer ratings and with global self-report measures of narcissism (Wink, 1992a). Hypersensitivity most closely resembles vulnerable narcissism and is the most clearly pathological (Pincus \& Lukowitsky, 2010). Hypersensitive individuals are characterized by grandiosity and entitlement, but these attributes are masked by inhibition, defensiveness, and a lack of self-confidence (Wink, 1992b). Hypersensitive individuals experience more marital conflict and higher levels of depression (Wink, 1992a, 1992b), and their spouses report that they are more defensive, anxious, and bitter (Wink, 1991b). In addition, Hypersensitivity is the only subscale associated with scores on the Narcissistic Personality Disorders Scale (NPDS; Ashby, Lee, \& Duke, 1979), a clinical measure derived from work with NPD patients (Wink, 1992a).

Wink's willfulness dimension most closely resembles grandiose narcissism (Pincus \& Lukowitsky, 2010). This dimension is characterized by exhibitionism, need for power, poor impulse control, and a pleasure-seeking orientation to life (Wink, 1992b). Willfulness encompasses a mixture of maladaptive and adaptive qualities, which are further reflected in its correlates: aggression, exhibitionism, and impulsivity, but also assertiveness, sociability, self-confidence, and psychological adjustment (Cramer \& Jones, 2008; Wink, 1992a, 1992b).

Finally, Wink's autonomy dimension encompasses the most adaptive aspects of narcissism, including self-reliance, selfdirectedness, psychological mindedness, and creativity (Wink, 1992b). Autonomous narcissism has been associated with good psychological health, greater empathy and self-control, and lower levels of depression (Cramer \& Jones, 2008; Wink, 1996). However, autonomy is also associated with aggression, exhibitionism, mania, and lack of self-control (Wink, 1992a), perhaps reflecting excessively high levels of agency (see also Russ et al., 2008). Thus, although autonomy predicts a number of positive outcomes, it also has some potentially maladaptive consequences. It is also important to note that scores on autonomy and willfulness tend to be positively correlated (Wink, 1992a), and, indeed, these two constructs share a number of correlates (e.g., aggression, exhibitionism). Correlations 
between these two constructs and hypersensitivity are generally modest, but autonomy is typically positively correlated with hypersensitivity and willfulness is typically negatively correlated with hypersensitivity (Cramer \& Jones, 2008; Wink, 1992a).

Based on prior work with similar samples (Wink, 1996), we expected that women in the current study would be characterized by higher levels of autonomy compared to hypersensitivity and willfulness; that is, on average, observer ratings would reveal fewer pathological and more adaptive aspects of narcissism. As described next, we also examined the extent to which these different aspects of narcissism changed as women aged from their 40 s to their 50 s.

\section{Longitudinal Changes in Narcissism}

There are several reasons to expect mean-level changes in narcissistic traits in middle adulthood, particularly for the more pathological aspects of this personality construct. Roberts et al. (2010), for instance, argued that narcissism should decrease with age because narcissistic traits are incompatible with normative developmental tasks of adulthood, such as the establishment of close relationships. People's opportunities to experience failure or adversity are also likely to increase with age, providing a kind of "reality check" on narcissistic tendencies (Foster et al., 2003). Additionally, as described earlier, with the transition to middle adulthood, narcissistic individuals may be forced to face their own limitations and impending mortality (Greenberg, 2009), potentially decreasing narcissism. Finally, pathological levels of narcissism can lead to significant impairments in important life domains, such as work and interpersonal relationships (Miller et al., 2007; Russ et al., 2008), which could ultimately interfere with a narcissist's ability to maintain his or her sense of grandiosity (Horowitz, 2009).

Although very few studies have examined age-related differences in narcissism, extant findings are consistent with the idea that narcissism decreases with age. Foster et al. (2003), for instance, examined cross-sectional differences in narcissistic traits (measured with the NPI) in a large sample of participants ranging in age from 8 to 83 . NPI scores were negatively correlated with age, and age-related decreases were largest for the most maladaptive subscales (i.e., exhibitionism, exploitativeness, and entitlement). Another crosssectional study of adults similarly found that overall NPI scores decreased with age (Roberts et al., 2010). 
Research with clinical populations also shows evidence of agerelated declines in narcissism. For instance, Rataj (2004) used a variety of measures to assess different aspects of narcissism in a cross-sectional sample of adults (ages 35 to 88 ) receiving psychiatric treatment. Narcissism scores generally decreased with age, particularly for more pathological aspects of narcissism, and this decline was most prominent beginning in the early 50s. Finally, in a 3-year longitudinal study of individuals diagnosed with NPD (ages 17 to 45 at the initial assessment), overall levels of pathological narcissism decreased over time (Ronningstam et al., 1995).

Taken together, these findings are consistent with the idea that narcissism declines in adulthood, with the most pathological aspects of narcissism showing the most change. However, because most data were obtained from cross-sectional studies, it is unclear whether age differences reflect normative developmental changes over time or cohort effects (e.g., Twenge, Konrath, Foster, Campbell, \& Bushman, 2008). Cross-sectional data also cannot answer questions about whether individual differences in narcissism are maintained over time despite mean-level change. We are aware of only one study that has assessed longitudinal changes in narcissistic traits from early through middle adulthood (Roberts \& Helson, 1997); however, this study reported correlations between changes in narcissism and other outcomes rather than absolute measures of change.

In the current study, we had the opportunity to examine both rank-order and mean-level changes in women's narcissism during middle adulthood. Given that personality typically shows high rank-order stability in adulthood (Roberts \& DelVecchio, 2000), we expected to find evidence for such stability of narcissism in our sample. Yet we also expected to find mean-level decreases in narcissism, particularly hypersensitive narcissism, from age 43 to 53 .

\section{Relations Between Narcissism and Women's Outcomes}

Based on prior work, we expected that hypersensitive narcissism would predict the most negative outcomes among women during the transition to middle adulthood, including more depressive symptoms, lower life satisfaction, and lower well-being. In contrast, we expected that autonomous narcissism would predict fewer negative outcomes and more positive outcomes, and that willful narcissism would predict a combination of negative and positive outcomes. 
In addition, although research on narcissism has focused almost exclusively on psychological outcomes, there are reasons to expect narcissism to predict poor physical health as well. Specifically, much narcissistic behavior can be characterized as defensive attempts to maintain unrealistically positive self-views (Morf \& Rhodewalt, 2001), and chronic reliance on such strategies has been associated with adverse physiological and health outcomes (e.g., Rutledge, 2006). Moreover, narcissistic traits have been linked with heightened physiological reactivity (Edelstein, Yim, \& Quas, 2010; Kelsey, Ornduff, McCann, \& Reiff, 2001), and, consistent with work on psychological outcomes, more maladaptive aspects of narcissism appear to be most closely associated with physiological dysregulation (Sommer, Kirkland, Newman, Estrella, \& Andreassi, 2009). Such dysregulation could have adverse implications for physical health over time (e.g., Chrousos \& Gold, 1992). Thus, we expected that hypersensitive narcissism would predict adverse physical as well as psychological health outcomes.

Finally, we expected increases in hypersensitivity from age 43 to 53 to predict poorer health and well-being outcomes at age 53 but increases in autonomy and willfulness to predict more positive outcomes and a combination of positive and negative outcomes, respectively.

\section{METHOD}

\section{Participants}

Participants were part of a larger longitudinal study of members of the Radcliffe College graduating class of 1964 (see Stewart \& Vandewater, 1993, for a more complete sample description). Narcissism measures were obtained from observer ratings, based on information provided at the 1986 and 1996 assessments, when the women were approximately 43 and 53 years old, respectively. The current analyses focus on the 70 women who completed both assessments. Mental health measures were completed at both time points; physical health and well-being measures were completed at the age 53 assessment. ${ }^{1}$ All but one woman was European American. Women had an average annual personal income of $\$ 40,000$ $\$ 50,000$ and an average annual household income of $\$ 100,000-\$ 120,000$.

1. A few participants were missing data for some of the outcome measures (see $N \mathrm{~s}$ in Table 1) and were not included in analyses involving those measures. 
Eighty percent had completed some graduate-level education. By age 53, $91 \%$ of the women had been married and $80 \%$ had children. The 70 women who completed both assessments did not significantly differ from those who did not on any of these demographic variables, narcissism, or mental health.

\section{Measures}

Narcissism was assessed at age 43 and 53 using the scales developed by Wink (1992a) for the California Adult Q-Sort (CAQ; Block, 1961). The CAQ consists of 100 descriptive items, which are sorted by trained observers into nine forced-choice categories, ranging from 1 (extremely uncharacteristic) to 9 (extremely characteristic). CAQ items cover a wide range of personality attributes, such as "Is vulnerable to real or fancied threat, generally fearful," "Is cheerful," and "Enjoys esthetic impressions; is esthetically reactive." The goal of the q-sort process is for raters to create descriptions of target individuals by achieving a consensual description of each target's personality (see Block, 2008). In the current study, three independent raters used the interview transcripts from the age 43 and 53 assessments to sort the CAQ items for participants at each time point. Raters had access to extensive material for each individual, including responses to open-ended questions and questions about interpersonal relationships, family, careers, life events, and future plans. These questions included the following: "When you think about the roles and identities that make you who you are, which ones stand out as being important to you, and why?"; "If you are remarried, how is your current marriage different from your previous marriage(s)?"; and "If you could do anything you wished in the next 10 years, what would you do?" Q-sort raters did not have access to closed-ended questionnaire data used as dependent measures in this study, and in general there was relatively little conceptual overlap between the information used to complete the q-sort and that included in these questionnaires.

Data were composited across raters, yielding an average score for each participant on each of the 100 items (as outlined in Block, 2008). Interrater reliability was typical of q-sort studies using a similar procedure: For the three raters of the 1986 (age 43) data, inter-rater agreement ranged from .60 to $.91(M=.77)$; inter-rater agreement for the three raters of the 1996 (age 53) data ranged from .60 to $.89(M=.78)$. A subset of CAQ items was then used to create the narcissism scales developed by Wink (1992a): The 12-item Hypersensitivity scale assesses defensive narcissism and includes items such as "is thin-skinned; sensitive to anything that can be construed as criticism or an interpersonal slight" and "concerned with own adequacy as a person, either at conscious or unconscious levels"; the 10-item Willfulness scale assesses more grandiose aspects of narcissism 
and includes items such as "is self-indulgent," "is self-dramatizing, histrionic," and "has a readiness to feel guilt" (contraindicative); and the 11-item Autonomy scale assesses "healthy" narcissism and is characterized by items such as "values own independence and autonomy" and "genuinely submissive; accepts domination comfortably" (contraindicative). The reliability and validity of these scales has been demonstrated by Wink (1992a, 1992b). Internal consistencies for these and all other measures are presented in Table 1.

Depressive symptoms were assessed by self-report at ages 43 and 53 using Zung's (1965) 20-item Depression Scale. Items assess depression severity, including symptoms such as crying spells, sleep disorders, and irritability. Participants rated each item on a scale ranging from 1 (never) to 4 (nearly all the time). Scores were derived by summing responses across items and then dividing the total score by 80 , resulting in a possible range from 0 to 1 .

Physical health problems were assessed by self-report at age 53 with three measures. The first was a set of 65 items adapted from the physical health section of the Cornell Medical Index (CMI; Brodman, Erdmann, Lorge, \& Wolff, 1949), a self-report symptom checklist designed to provide a broad assessment of physical health status, with questions pertaining to major physical systems (e.g., circulatory, respiratory). Participants were asked to rate the extent to which each item was applicable to them, using a 4-point scale ranging from 1 (never, or a little of the time true) to 4 (most often or always true), $M=1.35, S D=.17$. The second measure was a checklist of 17 chronic health conditions (e.g., asthma, hypertension, ulcers, migraines). Participants were asked to indicate whether they had any of these conditions, and the total number of items endorsed was used as a measure of chronic health problems, $M=1.30$, $S D=1.18$. The third measure was a single item that asked participants to rate their general state of health in the last 12 months, ranging from 1 (poor) to 5 (excellent), $M=4.29, S D=.92$. A composite index of physical health was created by averaging the three standardized measures. (The last measure was reverse-scored so that higher scores indicated more health problems.)

Psychological well-being was assessed by self-report at age 53 using the shortened (18-item) version of Ryff's original (1989) well-being measure (Ryff \& Keyes, 1995). Participants were asked to rate the extent to which they agreed with a series of statements (e.g., "I like most aspects of my personality") on a scale ranging from 1 (strongly disagree) to 6 (strongly agree), and items were averaged to create a total score.

Life satisfaction was assessed by self-report at age 53 using the Satisfaction with Life Scale (SWLS; Diener, Emmons, Larsen, \& Griffin, 1985), a five-item measure that includes items such as "In most ways my 


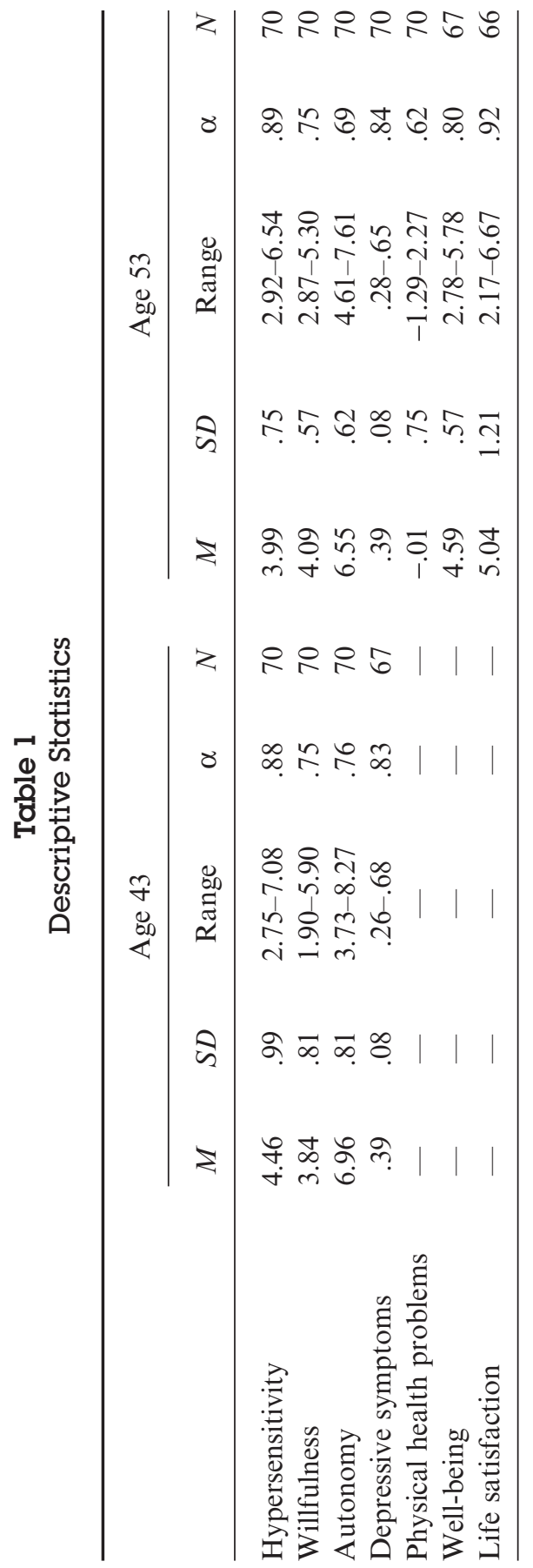


life is ideal." Items were rated on a 7-point scale ranging from 1 (strongly disagree) to 7 (strongly agree) and were averaged to create a total score.

\section{RESULTS}

Preliminary Analyses

Descriptive statistics for the primary study variables are presented in Table 1, and intercorrelations among the narcissism subscales are shown in Table 2. Consistent with prior research, autonomy and willfulness were positively correlated at both time points, but these subscales were not significantly correlated with hypersensitivity at either point.

\section{Changes in Narcissism Over Time}

Rank-order stability. We hypothesized that observer ratings of women's narcissism would show significant rank-order stability from age 43 to age 53. To test this hypothesis, we computed correlations between the age 43 and 53 scores for each subscale. As shown in Table 2, all three subscales showed significant stability over the 10 -year period, suggesting that women maintained their relative standing on the three dimensions of narcissism over time. Moreover, hypersensitivity demonstrated significantly more stability than willfulness, $Z=2.33, p<.05$, and marginally more stability than autonomy, $Z=1.67, p<.10$. The stability coefficients for willfulness and autonomy were not significantly different, $Z=-.72, p=.47$.

Mean-level change. We hypothesized that women in our sample would show higher levels of autonomy compared to hypersensitivity and willfulness at both time points. We also expected that women's levels of narcissism would decrease over time, and that such decreases would be most evident for hypersensitivity. To test these hypotheses, we conducted a repeated-measures analysis of variance (ANOVA) with narcissism subscale (hypersensitivity, willfulness, autonomy) and time (age 43, age 53) as within-subjects factors. Means from this analysis are shown in Table 1.

Results yielded a significant main effect of subscale, $F(2$, $138)=410.54, p<.01, \eta_{p}{ }^{2}=.86$, a main effect of time, $F(1$, 


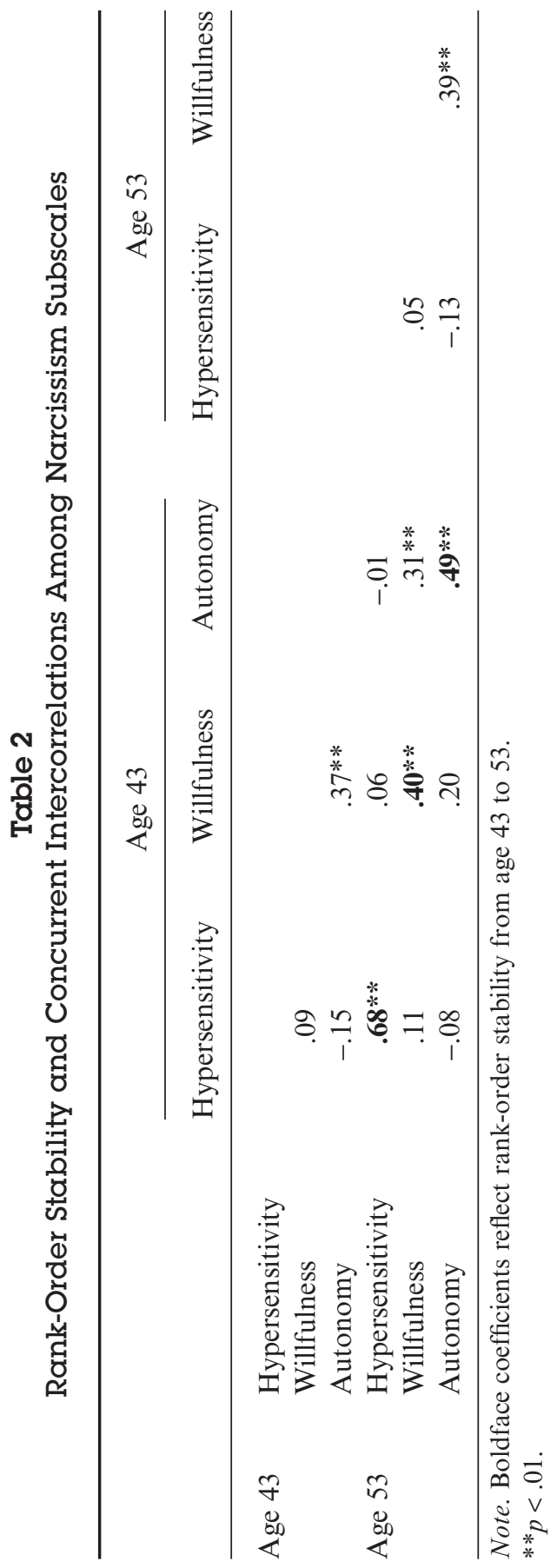


69) $=16.93, p<.01, \eta_{p}{ }^{2}=.20$, and an interaction between subscale and time, $F(2,138)=19.58, p<.01, \eta_{p}{ }^{2}=.22$. Consistent with our hypotheses, women showed higher levels of autonomy compared to hypersensitivity at age $43, t(69)=15.20, p<.01, d=1.83$, and at age $53, t(69)=20.69, p<.01, d=2.49$. Autonomy was also higher than willfulness at age $43, t(69)=28.64, p<.01, d=3.45$, and at age 53, $t(69)=31.20, p<.01, d=3.76$. In addition, hypersensitivity was significantly higher than willfulness at age $43, t(69)=4.24, p<.01$, $d=.51$, but scores on these two dimensions did not differ at age 53, $t(69)=-.89, p=.38, d=-.11$. Finally, all three subscales showed significant mean-level change over the 10-year period: As expected, there were decreases in hypersensitivity, $t(69)=-5.39, p<.01$, $d=-.96$, and in autonomy, $t(69)=-4.66, p<.01, d=-.81$, but there was also an unexpected (albeit smaller) increase in willfulness, $t(69)=2.61, p<.05, d=.46$.

Thus, women's levels of narcissism showed evidence of both rankorder stability and mean-level change. As expected, average levels of both hypersensitivity and autonomy decreased over the 10-year period. However, women also showed a small mean increase in willfulness, a subscale that includes both adaptive and maladaptive characteristics.

Relations Between Observer Ratings of Narcissism and Self-Reported Outcomes

Concurrent and prospective relations. We hypothesized that, of the three narcissism subscales, hypersensitivity would be the most consistent predictor of poor health and well-being outcomes. As shown in Table 3, hypersensitivity was in fact concurrently associated with more depressive symptoms, more physical health problems, lower well-being, and lower life satisfaction at both time points. The relations between hypersensitivity and these outcomes were also evident across the 10-year period (shown in the upper right quadrant of Table 3). Less consistent patterns emerged for willfulness and autonomy, although both subscales were negatively associated with concurrent depressive symptoms at age 43 and positively associated with concurrent well-being and life satisfaction at age 53 .

Because the subscales were intercorrelated, we also conducted regression analyses in which each outcome was predicted from all three subscales. Betas from these analyses, presented in Table 3, were 


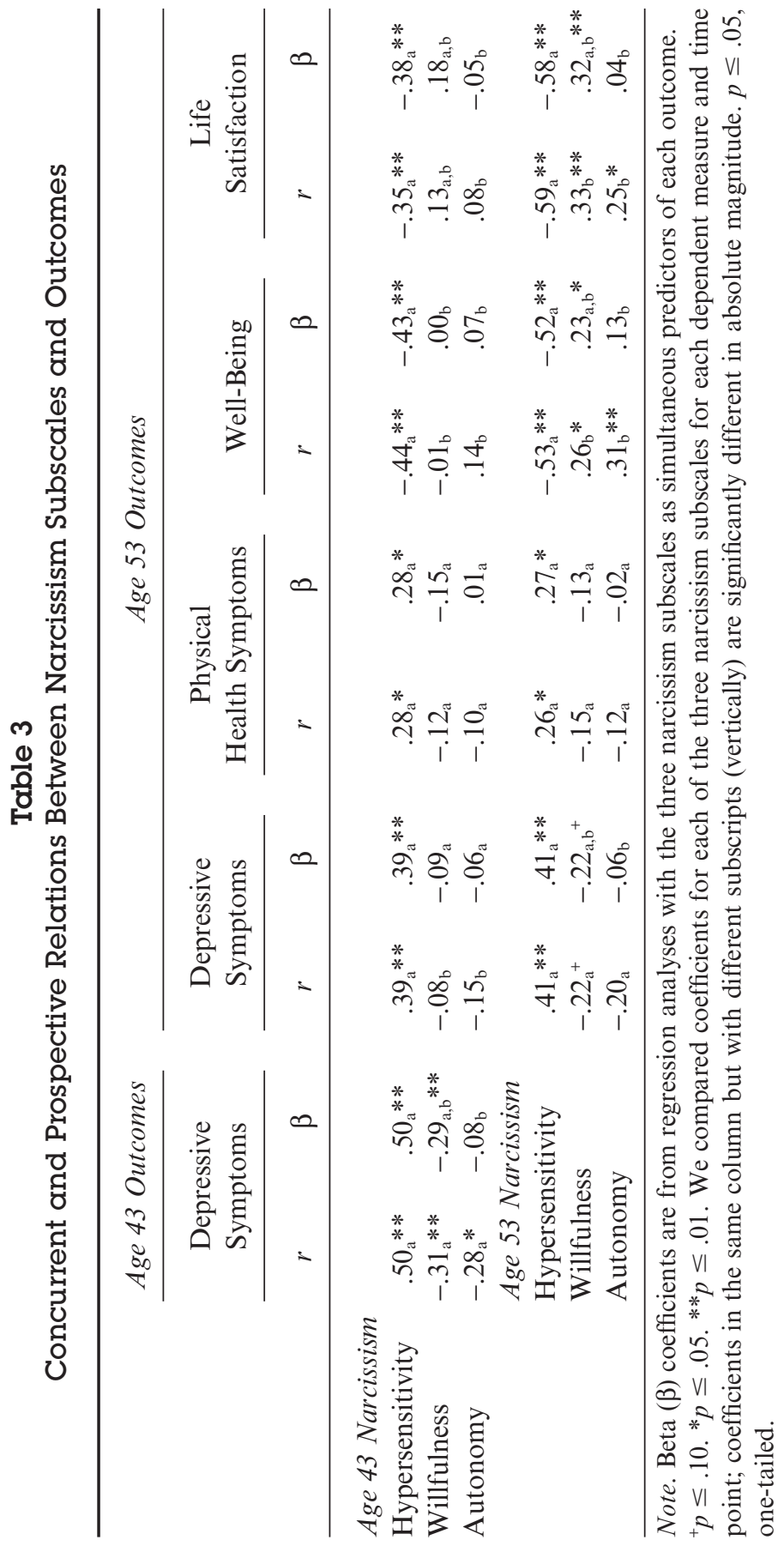


very similar to the zero-order correlations, with the exception that all of the associations between autonomy and our outcome measures became nonsignificant. These findings suggest that, in some cases, the associations between autonomy and the outcomes may have been due to the positive correlation between autonomy and willfulness.

Given our hypothesis that hypersensitivity would predict the poorest outcomes, we additionally tested whether this subscale was a stronger predictor of women's health and well-being compared to the other two subscales. We compared differences in absolute magnitude for the relevant bivariate and multivariate associations using onetailed tests of significance because we were specifically interested in whether the effects of hypersensitivity were larger than the effects of willfulness and autonomy. As shown in Table 3, for the majority of dependent measures, hypersensitivity was indeed a stronger predictor of women's outcomes compared to autonomy, and in many cases compared to willfulness.

Individual differences in change. We hypothesized that increases in hypersensitivity from age 43 to 53 would be associated with poor health and well-being at age 53, whereas increases in willfulness and autonomy would be associated with better health and well-being at age 53. To address questions about change over time, we computed residual change scores by regressing age 53 narcissism scores on age 43 scores and saving the residuals. Residual change scores provide a measure of how much each individual has changed and of the direction of that change; positive change scores indicate increases over time and negative scores indicate decreases over time. Unlike difference scores, residual change scores are statistically independent of initial status and therefore estimate change as if all individuals were at the same level at the initial assessment. ${ }^{2}$

Correlations between the residual change scores for the narcissism subscales and the age 53 health and well-being outcomes indicate whether changes in narcissism from age 43 to 53 predicted health and well-being at age 53. As shown in Table 4, increases in hypersensitivity predicted lower well-being and life satisfaction over the 10-year

2. One outcome measure, depressive symptoms, was also measured at both time points. However, depressive symptoms did not show any mean-level change over time (see Table 1), and residualized change scores for this measure were not significantly associated with any of the narcissism measures or with changes in narcissism, all $p \mathrm{~s}>.15$. 


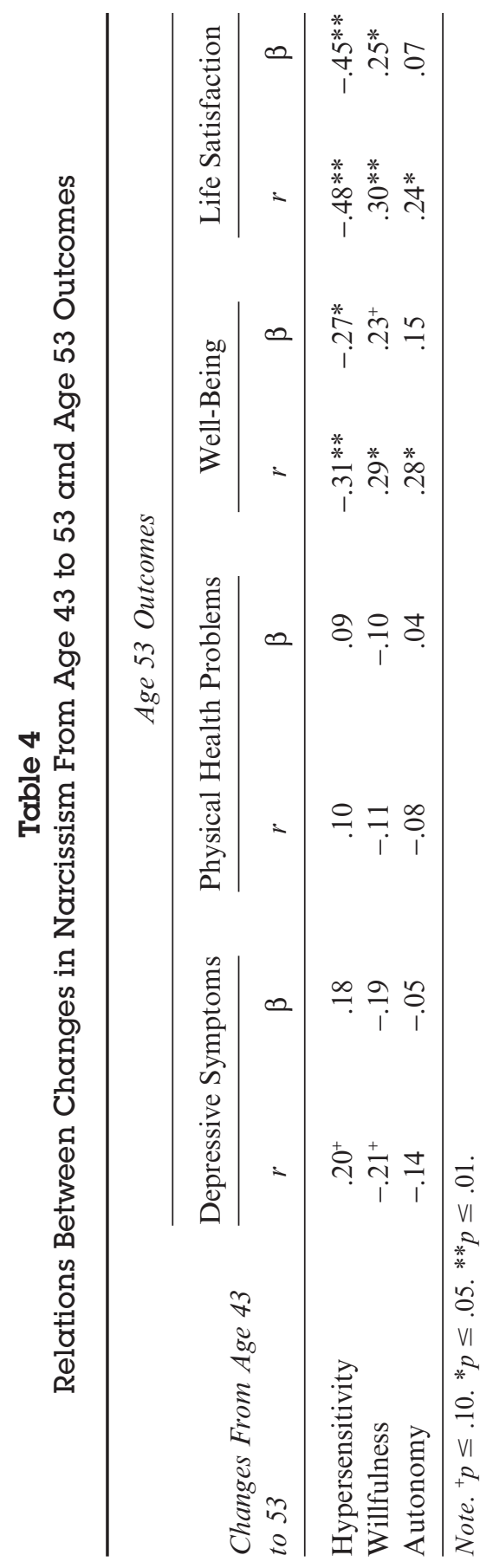


period, whereas increases in willfulness and autonomy predicted greater well-being and life satisfaction. Increases in willfulness and decreases in hypersensitivity were also marginally associated with fewer depressive symptoms at age 53. We additionally conducted regression analyses, in which each outcome was predicted from the residual change scores for the three narcissism subscales. Betas from these analyses, presented in Table 4, were very similar to the correlations, except that autonomy was no longer a significant predictor of change in either well-being or life satisfaction.

In summary, our analyses of individual differences in change indicated that women who increased in hypersensitivity from age 43 to 53 were less well adjusted and satisfied with their lives at age 53. In contrast, women who increased in willfulness and/or autonomy during this period were better adjusted and more satisfied with their lives at age 53 .

\section{DISCUSSION}

The current study examined the role of narcissistic personality traits in women's health and well-being during the transition to middle adulthood. Although this life stage is thought to be particularly difficult for narcissistic individuals (e.g., Kernberg, 1975), few studies have assessed the nature and correlates of narcissism in middle adulthood. In our sample of college-educated women, narcissism was associated with important health and well-being outcomes at ages 43 and 53, although the nature of these associations varied for different kinds of narcissism. Specifically, observer ratings of hypersensitive narcissism, thought to encompass the most pathological characteristics of this personality construct, were associated with more negative health and well-being outcomes. These relations were observed not only concurrently but also over a 10 -year period. Moreover, increases in hypersensitive narcissism from age 43 to 53 predicted lower well-being and life satisfaction at age 53, suggesting that changes in narcissism, in addition to absolute levels, have important implications for adjustment in middle adulthood.

Findings from the current study also contribute to a growing body of work on the physiological and health implications of narcissism. Previous research demonstrates that narcissism predicts greater physiological stress reactivity (Edelstein et al., 2010; Kelsey et al., 
2001), and that such effects are most evident for more pathological aspects of narcissism (Sommer et al., 2009). Insofar as these responses are experienced chronically, they could ultimately impact physical health (Chrousos \& Gold, 1992). Indeed, in our sample, higher levels of hypersensitive narcissism were associated with more concurrent physical health complaints at both time points, suggesting that the defensive nature of hypersensitivity might exact a physiological toll that could influence physical health (e.g., Rutledge, 2006). The correlational nature of our data precludes firm conclusions about the causal direction of these processes; however, future work could examine these hypotheses more directly by assessing chronic stress responses among narcissistic individuals and the extent to which such responses predict long-term outcomes. Moreover, physical health concerns typically become more prominent beginning in middle adulthood (e.g., Lachman, 2004), so middle and older adulthood may be especially opportune times to examine associations between narcissism and physical health outcomes.

Autonomy and willfulness were less consistent predictors of women's health and well-being, but in general these aspects of narcissism were associated with positive outcomes. Increases in autonomy and willfulness from age 43 to 53 also predicted greater well-being and life satisfaction at age 53. Of note, autonomy and willfulness were positively correlated in our sample, and when all three subscales were entered as simultaneous predictors, all of the positive relations between autonomy and women's outcomes became nonsignificant. Thus, our data suggest that autonomy may have relatively little predictive utility when considered along with willfulness, at least for the constructs that we measured in this study. Other studies similarly find that adaptive aspects of narcissism are less consistently associated with well-being and adjustment (e.g., Ackerman et al., 2011; Wink, 1991a). Moreover, although there is some disagreement about how best to conceptualize narcissism (e.g., Emmons, 1984; Miller et al., 2011), some have questioned whether "healthy" aspects of narcissism should even be considered part of this construct, particularly given its clinical roots and implications (Ackerman et al., 2011; Rosenthal \& Hooley, 2010). Although our data cannot address the structure of narcissism per se, our findings provide additional support for the centrality of grandiose and vulnerable aspects of narcissism for personality functioning (Miller et al., 2011; Pincus \& Lukowitsky, 2010). It is also interesting to note 
Wink's (1991a) finding that expert ratings of a "prototypical" narcissist were more highly correlated with hypersensitivity and willfulness than with autonomy.

In this light, the positive relations between willfulness and women's outcomes may seem even more surprising, given that willfulness has primarily negative elements, including grandiosity and poor impulse control. However, other aspects of willfulness could have positive implications, such as the ability to express hostile feelings directly and self-directedness. Moreover, prior studies have linked willfulness with positive outcomes that are similar to those measured in the current study, including lower levels of depression and greater psychological adjustment (Cramer \& Jones, 2008; Wink, 1992a, 1992b). Miller and colleagues (2011) also found that measures of "grandiose" narcissism, which shares many conceptual similarities to willfulness, had some positive correlates, such as extraversion and achievement.

It is also possible that the meaning of willfulness is tied to the life contexts of the women in our sample, who came of age during the 1960s, a time of dramatic changes in women's social roles (Chafe, 1974). Perhaps what observers perceived as "willfulness" could also be characterized as assertiveness or confidence. In fact, Neugarten (1968) hypothesized that "executive personality," exemplified by feelings of mastery and competence, increased during middle age for both men and women. Moreover, other longitudinal studies of women during midlife and from similar cohorts have documented increases in personality constructs that share these aspects of willfulness (e.g., dominance, decisiveness, confident power; Helson, Jones, \& Kwan, 2002; Stewart et al., 2001). Thus, certain aspects of willful narcissism may reflect normative changes in women's personality during middle adulthood, at least for women in this particular cohort, and such changes appear to be associated with positive development.

Our findings also contribute to research on the developmental course of narcissistic personality traits. Although there are reasons to expect that levels of narcissism, particularly hypersensitivity, would decrease across the life span (e.g., Kernberg, 1975), to our knowledge, our study is the first to report longitudinal changes in narcissistic traits in middle adulthood. We found that, as a group, the women in our sample were characterized by high levels of autonomy and relatively low levels of willfulness and hypersensitivity. These 
mean-level differences are similar to those reported by Wink (1996) in another sample of college-educated women. Interestingly, Wink (1996) similarly found that, although most women were characterized as autonomous, more women were characterized as hypersensitive than willful at age 43 . The difference between hypersensitivity and willfulness was relatively small in our study at age 43, and nonexistent at age 53, perhaps reflecting a developmental transition away from more vulnerable expressions of narcissism, which may be associated with positive outcomes.

Moreover, all three narcissism subscales showed significant rankorder consistency from age 43 to 53, indicating that women generally maintained their standing on these subscales relative to other participants in the sample. Women's scores on hypersensitivity were particularly stable over time. Perhaps, despite normative developmental changes, hypersensitivity is less sensitive to contextual influences compared to willfulness and autonomy. The high levels of stability found in the current study are consistent with other research on longitudinal changes in personality (Roberts \& DelVecchio, 2000). They are also particularly noteworthy given that women's narcissism scores were derived from observer ratings of open-ended interview responses, rather than from self-report measures.

At the same time, all three subscales showed significant mean-level change, including hypothesized decreases in hypersensitivity and autonomy. We also found an unexpected increase in willfulness, although willfulness showed the smallest amount of change compared to the other two subscales. In addition, as described earlier, it is possible that at least some aspects of willfulness reflect positive aspects of women's development, particularly in this cohort. Unfortunately, we only had information on women's narcissism at age 43 and 53, so it is not clear how changes during this time period compare to those that might be observed in adolescence, early adulthood, or even old age. For instance, our data cannot address questions about when in adulthood narcissism begins to decline, whether this decline continues throughout adulthood, or whether stability increases across the life span.

More generally, it is important to consider our findings in light of the unique characteristics of our sample. Although our study provided a rare opportunity to examine changes in narcissism over a 10 -year period, it is nonetheless a relatively small and homogeneous sample. The women in this study are high-achieving, almost exclu- 
sively Caucasian graduates from an elite liberal arts college. This represents an important limitation with respect to the generalizability of our findings, and it will be important to investigate whether our findings replicate in more diverse samples of women or among men. Although men typically score somewhat higher on self-report narcissism measures, such as the NPI (e.g., Foster et al., 2003), and on clinical measures of NPD symptomatology (Miller et al., 2007), some have argued that women are more likely to display hypersensitive compared to willful aspects of narcissism (e.g., O'Leary \& Wright, 1986). Thus, future research should assess whether gender moderates any of the associations that we found in this middle-aged sample.

It is also important to note that our measure of narcissism was derived from observer ratings rather than from self-report. We view this as an important strength of our study because it decreases any shared method variance between narcissism and women's selfreported outcomes. However, it is possible that observers were unable to capture some aspects of narcissism that are more internal or less amenable to observation (e.g., self-presentation concerns). Indeed, narcissistic traits are more typically measured via self-report, and there is some evidence that hypersensitive narcissism may be more difficult for observers to detect than willful narcissism (Wink, 1991b). At the same time, self- and other ratings of trait narcissism show a relatively high degree of convergence (e.g., correlations around .40; Wink, 1992a), even following relatively brief interactions (Vazire, Naumann, Rentfrow, \& Gosling, 2008), suggesting that observers can evaluate narcissism in others with at least some degree of accuracy. Research on NPD similarly suggests that there is consensus among peer ratings of pathological narcissism, but considerable discordance between other ratings and self-ratings (Pincus \& Lukowitsky, 2010). Ultimately, because narcissism is characterized by a lack of insight into one's own behavior (Kernberg, 1975), it will be important in future research to use multiple measures of this personality construct, and it will be interesting to explore whether and how the correlates of self-reported narcissism differ from those of observer-rated narcissism.

Finally, although our theoretical framework conceptualizes narcissism as a precursor of or contributor to women's outcomes, it is also possible that the causal direction works in the opposite way. That is, poor health and well-being may increase hypersensitive nar- 
cissism and decrease willful and/or autonomous narcissism over time. It is also possible that other unmeasured variables may partially explain the associations that we found in the current study. For instance, broader temperamental characteristics or motivational profiles might predict both narcissism and health and well-being outcomes. The prospective relations that we uncovered and our analyses of change over time bolster our interpretations about causality, but only more comprehensive longitudinal research can more fully address these kinds of questions.

Despite these limitations, our findings make important contributions to the literature on narcissism and personality development by documenting longitudinal changes in narcissism and demonstrating that different aspects of narcissism predict different life outcomes during the transition to middle adulthood. All three aspects of narcissism showed considerable rank-order stability across the 10-year period. Yet hypersensitive narcissism decreased over time and was associated with the most negative outcomes for women, whereas willful narcissism increased and was associated with the most positive outcomes. Autonomous narcissism showed a less consistent pattern of findings: Levels of this personality construct decreased from age 43 to 53, and predicted some positive outcomes at both time points, but autonomy did not show any independent associations with women's outcomes when the other subscales were simultaneously considered. Taken together, our findings highlight the utility of measuring different aspects of narcissism in relation to women's outcomes and patterns of development during middle adulthood.

\section{REFERENCES}

Ackerman, R. A., Witt, E. A., Donnellan, M. B., Trzesniewski, K. H., Robins, R. W., \& Kashy, D. A. (2011). What does the Narcissistic Personality Inventory really measure? Assessment, 18, 67-87.

American Psychiatric Association. (1994). Diagnostic and statistical manual of mental disorders (4th ed.). Washington, DC: Author.

Ashby, H. U., Lee, R. R., \& Duke, E. H. (1979). A narcissistic personality disorder MMPI scale. Paper presented at the annual meeting of the American Psychological Association, New York.

Block, J. (1961). The q-sort method in personality assessment and psychiatric research. Springfield, IL: Charles C. Thomas.

Block, J. (2008). The q-sort in character appraisal: Encoding subjective impressions of persons quantitatively. Washington, DC: American Psychological Association. 
Brodman, K., Erdmann, A., Jr., Lorge, I., \& Wolff, H. (1949). The Cornell Medical Index: An adjunct to medical interview. Journal of the American Medical Association, 140, 530-534.

Cain, N. M., Pincus, A. L., \& Ansell, E. B. (2008). Narcissism at the crossroads: Phenotypic description of pathological narcissism across clinical theory, social/ personality psychology, and psychiatric diagnosis. Clinical Psychology Review, 28, 638-656.

Chafe, W. (1974). The American woman: Her changing social, economic, and political roles, 1920-1970. New York: Oxford University Press.

Chrousos, G. P., \& Gold, P. W. (1992). The concepts of stress and stress systems disorders: Overview of physical and behavioral homeostasis. Journal of the American Medical Association, 267, 1244-1252.

Cramer, P., \& Jones, C. J. (2008). Narcissism, identification, and longitudinal change in psychological health: Dynamic predictions. Journal of Research in Personality, 42, 1148-1159.

Diener, E., Emmons, R. A., Larsen, R. J., \& Griffin, S. (1985). The Satisfaction With Life Scale. Journal of Personality Assessment, 49, 71-75.

Edelstein, R. S., Yim, I. S., \& Quas, J. A. (2010). Narcissism predicts heightened cortisol reactivity to a psychosocial stressor in men. Journal of Research in Personality, 44, 565-572.

Emmons, R. A. (1984). Factor analysis and construct validity of the Narcissistic Personality Inventory. Journal of Personality Assessment, 48, 291-300.

Foster, J. D., Campbell, W. K., \& Twenge, J. M. (2003). Individual differences in narcissism: Inflated self-views across the lifespan and around the world. Journal of Research in Personality, 37, 469-486.

Greenberg, T. M. (2009). Narcissistic aspects of aging and illness. In T. M. Greenberg (Ed.), Psychodynamic perspectives on aging and illness (pp. 59-74). New York: Springer.

Helson, R., Jones, C., \& Kwan, V. S. Y. (2002). Personality change over 40 years of adulthood: Hierarchical linear modeling analyses of two longitudinal samples. Journal of Personality and Social Psychology, 83, 752766.

Helson, R., \& Wink, P. (1992). Personality change in women from the early 40s to the early 50s. Psychology and Aging, 7, 46-55.

Horowitz, M. (2009). Clinical phenomenology of narcissistic pathology. Psychiatric Annals, 39, 124-128.

Kelsey, R. M., Ornduff, S. R., McCann, C. M., \& Reiff, S. (2001). Psychophysiological characteristics of narcissism during active and passive coping. Psychophysiology, 38, 292-303.

Kernberg, O. F. (1975). Borderline conditions and pathological narcissism. New York: Jason Aronson.

Kohut, H. (1971). The analysis of the self. New York: International University Press.

Kohut, H. (1977). The restoration of the self. New York: International University Press.

Lachman, M. E. (2004). Development in midlife. Annual Review of Psychology, 55, 305-331. 
Miller, J. D., \& Campbell, W. K. (2010). The case for using research on trait narcissism as a building block for understanding narcissistic personality disorder. Personality Disorders: Theory, Research, and Treatment, 1, 180-191.

Miller, J. D., Campbell, W. K., \& Pilkonis, P. A. (2007). Narcissistic personality disorder: Relations with distress and functional impairment. Comprehensive Psychiatry, 48, 170-177.

Miller, J. D., Hoffman, B. J., Gaughan, E., Gentile, B., Maples, J., \& Campbell, W. K. (2011). Grandiose and vulnerable narcissism: A nomological network analysis. Journal of Personality, 79, 1013-1042.

Morf, C. C., \& Rhodewalt, F. (2001). Unraveling the paradoxes of narcissism: A dynamic self-regulatory processing model. Psychological Inquiry, 12, 177196.

Neugarten, B. L. (1968). The awareness of middle age. In B. L. Neugarten (Ed.), Middle age and aging (pp. 93-98). Chicago: University of Chicago Press.

O'Leary, J., \& Wright, F. (1986). Shame and gender issues in pathological narcissism. Psychoanalytic Psychology, 3, 327-339.

Pincus, A. L., \& Lukowitsky, M. R. (2010). Pathological narcissism and narcissistic personality disorder. Annual Review of Clinical Psychology, 6, 421446.

Raskin, R., \& Hall, C. S. (1979). A narcissistic personality inventory. Psychological Reports, 45, 590.

Rataj, D. M. (2004). Changes in pathological narcissism from midlife to older adulthood. Unpublished doctoral dissertation, Northwestern University.

Roberts, B. W., \& DelVecchio, W. (2000). The rank-order consistency of personality traits from childhood to old age: A quantitative review of longitudinal studies. Psychological Bulletin, 126, 3-25.

Roberts, B. W., Edmonds, G., \& Grijalva, E. (2010). It is developmental me, not generation me: Developmental changes are more important than generational changes in narcissism. Perspectives on Psychological Science, 5, 97-102.

Roberts, B. W., \& Helson, R. (1997). Changes in culture, changes in personality: The influence of individualism in a longitudinal study of women. Journal of Personality and Social Psychology, 72, 641-651.

Roberts, B. W., Walton, K., \& Viechtbauer, W. (2006). Patterns of mean-level change in personality traits across the life course: A meta-analysis of longitudinal studies. Psychological Bulletin, 132, 1-25.

Ronningstam, E., Gunderson, J., \& Lyons, M. (1995). Changes in pathological narcissism. American Journal of Psychiatry, 152, 253-257.

Rosenthal, S. A., \& Hooley, J. M. (2010). Narcissism assessment in socialpersonality research: Does the association between narcissism and psychological health result from a confound with self-esteem? Journal of Research in Personality, 44, 453-465.

Russ, E., Shedler, J., Bradley, R., \& Westen, D. (2008). Refining the construct of narcissistic personality disorder: Diagnostic criteria and subtypes. American Journal of Psychiatry, 165, 1473-1481.

Rutledge, T. (2006). Defensive personality effects on cardiovascular health: A review of the evidence. In D. Johns (Ed.), Stress and its impact on society (pp. 1-21). Hauppauge, NY: Nova Science. 
Ryff, C. D. (1989). Happiness is everything, or is it? Explorations on the meaning of psychological well-being. Journal of Personality and Social Psychology, 57, 1069-1081.

Ryff, C. D., \& Keyes, C. L. M. (1995). The structure of psychological well-being revisited. Journal of Personality and Social Psychology, 69, 719-727.

Sommer, K. L., Kirkland, K. L., Newman, S. R., Estrella, P., \& Andreassi, J. L. (2009). Narcissism and cardiovascular reactivity to rejection imagery. Journal of Applied Social Psychology, 39, 1083-1115.

Stewart, A. J., Ostrove, J. M., \& Helson, R. (2001). Middle aging in women: Patterns of personality change from the 30 s to the 50s. Journal of Adult Development, 8, 23-37.

Stewart, A. J., \& Vandewater, E. A. (1993). The Radcliffe class of 1964: Career and family social clock projects in a transitional cohort. In K. D. Hulbert \& D. T. Schuster (Eds.), Women's lives through time: Educated American women of the twentieth century (pp. 235-258). San Francisco: Jossey-Bass.

Stewart, A. J., \& Vandewater, E. A. (1999). "If I had it to do over again . .": Midlife review, midcourse corrections, and women's well-being in midlife. Journal of Personality and Social Psychology, 76, 270-283.

Twenge, J. M., Konrath, S., Foster, J. D., Campbell, W. K., \& Bushman, B. J. (2008). Egos inflating over time: A cross-temporal meta-analysis of the Narcissistic Personality Inventory. Journal of Personality, 76, 875-902.

Vazire, S., Naumann, L. P., Rentfrow, P. J., \& Gosling, S. D. (2008). Portrait of a narcissist: Manifestations of narcissism in physical appearance. Journal of Research in Personality, 42, 1439-1447.

Wink, P. (1991a). Self- and object-directedness in middle-aged women. Journal of Personality, 59, 769-791.

Wink, P. (1991b). Two faces of narcissism. Journal of Personality and Social Psychology, 61, 590-597.

Wink, P. (1992a). Three narcissism scales for the California Q-set. Journal of Personality Assessment, 58, 51-66.

Wink, P. (1992b). Three types of narcissism in women from college to mid-life. Journal of Personality, 60, 7-30.

Wink, P. (1996). Transition from the early 40s to the early 50s in self-directed women. Journal of Personality, 64, 49-70.

Zuckerman, M., \& O'Loughun, R. E. (2009). Narcissism and well-being: A longitudinal perspective. European Journal of Social Psychology, 39, 957-972.

Zung, W. W. (1965). A self-rating depression scale. Archives of General Psychiatry, $12,63-70$. 